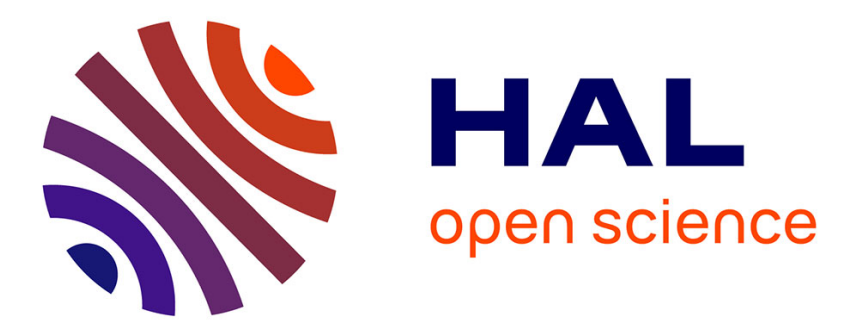

\title{
Classificateurs numéraux en purépecha : entre perte de vitalité et motivation pragmatique
}

Claudine Chamoreau

\section{To cite this version:}

Claudine Chamoreau. Classificateurs numéraux en purépecha : entre perte de vitalité et motivation pragmatique. Bulletin de la Société de Linguistique de Paris, 2013, 49 (2), pp.51-66. 10.3917/ling.492.0051 . halshs-01100138

\section{HAL Id: halshs-01100138 \\ https://shs.hal.science/halshs-01100138}

Submitted on 6 Jan 2015

HAL is a multi-disciplinary open access archive for the deposit and dissemination of scientific research documents, whether they are published or not. The documents may come from teaching and research institutions in France or abroad, or from public or private research centers.
L'archive ouverte pluridisciplinaire HAL, est destinée au dépôt et à la diffusion de documents scientifiques de niveau recherche, publiés ou non, émanant des établissements d'enseignement et de recherche français ou étrangers, des laboratoires publics ou privés. 


\title{
CLASSIFICATEURS NUMÉRAUX EN PUREPECHA : ENTRE PERTE DE VITALITE ET MOTIVATION PRAGMATIQUE
}

\author{
par Claudine CHAMOREAU \\ SeDyL-CELIA (CNRS)
}

El purepecha cuenta con una clase de tres elementos que han sido descritos como clasificadores numerales. Ocurren en una frase nominal con un numeral y dan informaciones semánticas sobre la característica física de la entidad expresada por el sustantivo (Chamoreau 1999, Friedrich 1970, 1984, Nava 1997, Vázquez Rojas 2012). El objetivo de este estudio es de describir la dinámica actual de estas unidades analizando los diferentes indicios que muestran su pérdida de vitalidad (reducción del inventario, erosión fonética, uso opcional, variaciones pragmáticas) y mostrando cómo el proceso de pérdida está relacionado con una motivación pragmática. Los clasificadores numerales aparecen siempre en un contexto en el que refieren a una entidad prominente e identifiable, accesible al hablante y al interlocutor:

\section{INTRODUCTION}

Le purepecha ${ }^{\mathbf{1}}$ possède une classe composée de trois éléments que l'on peut caractériser comme des classificateurs numéraux (désormais CNum) (Aikhenvald 2003, Grinevald 1999, 2000). Au sein du syntagme nominal (désormais $\mathrm{SN}$ ), ils se présentent toujours après le numéral et avant le nom. Comme nous pouvons observer dans les exemples (1), (2) et (3), ces unités renvoient à des particularités physiques des référents des entités modifiées : ichakwa renvoie à une forme longue, ichukwa, à une forme plate et irakwa, à une forme ronde.
(1) kama-h-ti
ma ichakwa tataka
élever-AOR-ASS3
un CNUM.long jeune-homme 'Il éleva un jeune-homme.'

1 Le purepecha, connu aussi sous le nom de tarasque, est une langue génétiquement isolée parlée au Mexique par environ 120000 personnes. 
(2) ixu ja-rha-s-ti

t’amu ichukwa ichuskuta

ici être.là-FT-AOR-ASS3 quatre CNUM.plat tortilla ${ }^{2}$

'Ici, il y a quatre tortillas.'

$\begin{array}{lll}\text { (3) yumu irakwa } & \text { kwaxanta=rini } & \text { intsku-s-ti } \\ \text { cinq CNUM.rond oeuf=1OBJ } & \text { donner-AOR-ASS3 } \\ \text { 'Il m'a donné cinq oeufs.' } & \end{array}$

En purepecha, les CNums ont généralement été décrits comme des unités qui apportent des informations sémantiques concernant la forme de l'unité quantifiée (Chamoreau 1999, Friedrich 1970, 1984, Nava 1997, Vázquez Rojas 2012). Au niveau lexical, les CNums sont formés à partir de bases qui expriment les caractéristiques physiques de l'élément référé et permettent de le localiser dans un espace. Comme nous pouvons l'observer dans l'exemple (4), la base kirá- 'rond' est choisie car elle renvoie à la forme de l'élément qui fonctionne comme sujet, la marmite, elle est suivie par le suffixe locatif $-n u$ qui indique sa localisation, le patio. Ces bases forment un ensemble de plus de trente unités qui ont été étudiées de façon précise (Capistrán 2000, Friedrich 1970, 1984).

\section{(4) tsúntsu kira-nu-s-ti \\ marmite rond-patio-AOR-ASS3 \\ 'La marmite est dans le patio.'}

L'objet de cette étude est de décrire la dynamique actuelle de ces unités en analysant les différents indices qui démontrent leur perte de vitalité (réduction de l'inventaire d'unités, érosion phonétique, utilisation optionnelle, variations sémantiques) et en montrant comment ce processus de perte est accompagné d'une motivation pragmatique. Les CNums sont rarement utilisés (moins de $7 \%$ des SNs avec un numéral), et le sont toujours dans un contexte marqué où ils renvoient à une entité prominente et identifiable, au sens de Lambrecht (1994 : 77-78), c'est-à-dire qu'elle constitue le premier plan de la situation, et est accessible au locuteur et à l'interlocuteur. Cette recherche démontre donc que les CNums apportent aussi des informations pragmatiques et

2 Au Mexique, la tortilla est une sorte de crêpe de maïs. 
n'expriment pas uniquement des caractéristiques sémantiques des unités référées.

Cet article s'organise de la façon suivante : après une brève introduction des caractéristiques générales du syntagme nominal avec un CNum dans la deuxième partie, la troisième exposera les différents indices qui montrent la perte de vitalité de ces unités. Finalement, la quatrième partie est consacrée à l'analyse pragmatique.

\section{CARACTÉRISTIQUES GÉNÉRALES DU SN AVEC UN CNUM}

Le purepecha est une langue dans laquelle le nom peut fonctionner comme le prédicat de l'énoncé. Il est alors généralement accompagné de l'unité - $e$, appelée prédicativisateur, comme dans l'exemple (5). Il n'existe pas de marquage en genre, en revanche le nom peut être modifié par le pluriel si son référent est animé ou individué (Chamoreau 2009, Chamoreau et Argueta 2011), comme dans les exemples (5) et (6). Dans la situation où a été prononcé cet énoncé, les deux noms renvoient à des entités multiples, mais seul iwiri 'bûcheron' est modifié par le pluriel car il est animé, en revanche anatapu 'arbre' conserve la même forme qu'au singulier car il renvoie à des entités inanimées et non individualisables dans cette situation. Le purepecha possède un article indéfini, ma, qui est une forme grammaticalisée du numéral ma 'un'. Comme on peut le constater dans l'énoncé en (5), ma présente le plus haut degré de grammaticalisation de l'échelle présentée par Heine (1997 : 70-76, 2012 : 134-139) puisqu'il peut modifier des noms au pluriel (Chamoreau 2012). S'il est modifié par un article indéfini, le nom au pluriel renvoie à un groupe d'entités non identifiables une à une, comme en (5).

(5) ma axuni-echa-e-s-ti=ksï

ART.INDEF cerf-PL-PRED-AOR-ASS $3=3$ PL

'Ce sont des cerfs.'

(6) iwiri-echa

p'iku-x-ti=kxï

anatapu

bûcheron-PL couper-AOR-ASS3=3PL arbre

'Les bûcherons coupèrent des arbres.' 
Les CNums apparaissent dans un $\mathrm{SN}$ dans lequel un numéral modifie un nom [Num CNum N], comme en (7). Ils peuvent aussi accompagner l'interrogatif namuni 'combien' [INT CNum N], comme en (8).

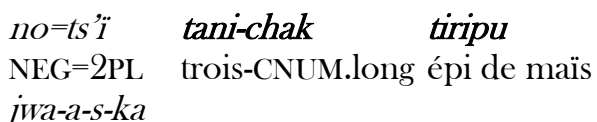

apporter-3PL.OBJ-AOR-ASS1/2

'Vous n'avez pas apporté trois épis de mais.'
(8) namuni-rhak mantsana ja-rha-s-ki
combien-CNUM.rond pomme être.là-FT-AOR-INT
'Combien de pommes y a-t-il?'

Le nom peut être absent du SN, [Num CNum], lorsque le CNum procède à une reprise anaphorique, comme on peut l'observer dans l'exemple (9b) :
(9)a. namuni=ri ichuskuta
combien $=2$ tortilla
pya-kuri-s-ki
'Combien de tortillas t'es-tu acheté?'

\section{b. tsiman-ichuk $=k^{\prime} u$ \\ deux-CNUM.plat=seulement \\ 'Seulement deux'}

Greenberg (1974 : 25) indique que les CNums n'apparaissent généralement pas avec des noms dont la pluralité est obligatoirement marquée, en revanche ils peuvent apparaître avec des noms dont la pluralité est indiquée de façon facultative. C'est le cas en purepecha : dans un GN avec un numéral et un CNum, le nom peut être modifié par le pluriel si son référent est animé, comme dans l'exemple (10). Si son référent est inanimé, la présence du pluriel indique une lecture individuée et définie (Chamoreau 2009 : 86-92, Chamoreau et Argueta 2011 : 104112), comme dans l'exemple (11a) qui s'oppose à (11b) où le nom n’est pas modifié par le pluriel, le GN est donc indéfini : 
(10) ima

tsimani echakwa

DEM

jinkoni

COM

deux CNUM.long

jama-s- $t i$

marcher-AOR-ASS3

'On dit qu'il marchait avec deux personnes.'

(11)a. jwa-chi-a-e=rini

tsimani-rhak

apporter-APPL1/2-3PL.OBJ-IMP.2=1OBJ deux-CNUM.rond naraxa-icha

orange-PL

'Apporte-moi les deux oranges.'

b. jwa-chi-e=rini apporter-APPL1/2-IMP.2=1OBJ cinq-CNUM.rond 'Apporte-moi cinq oranges.'

\section{INDICES DE PERTE DE VITALITÉ}

La perte de vitalité des CNums est considérable et s'est accélérée ces dernières années (voir un premier constat établi il y a 15 ans, Chamoreau 1999). Elle se manifeste à différents niveaux : la réduction du nombre des unités, passant de 18 éléments au XVIème siècle à 3 unités actuellement (3.1.), l'érosion phonétique qui a pour conséquence la perte de l'accent et la dépendance formelle des unités (3.2.), le caractère optionnel des CNums qui ne sont attestés, dans notre corpus, que dans $7 \%$ des $\mathrm{SNs}$ avec un numéral (3.3.), et finalement les différentes variations sémantiques qui ont pour conséquence l'utilisation croissante du CNum echawa, quel que soit le contexte ou la caractéristique physique du référent (3.4.).

\subsection{Réduction de l'inventaire des unités}

Les CNums décrits au XVIème siècle forment un ensemble de dix-huit éléments ${ }^{3}$. Dans la première colonne du tableau 1 sont

3 On peut s'interroger comme le fait, de façon pertinente, Nava (1997) sur le nombre exact de ces unités et sur leur utilisation exacte au XVIème siècle. 
regroupées les formes telles qu'elles sont transcrites par Gilberti (1987 [1558] : 275). La deuxième colonne indique le sens des unités tel qu'il est donné par Gilberti et précisé par Lagunas 1983 [1574] : 168-169). Cet auteur a repris l'inventaire de Gilberti, en respectant l'ordre mais en précisant le sens de certains éléments.

\section{Tableau 1. Formes utilisées au XVIème siècle}

\begin{tabular}{|c|c|}
\hline Formes & Sens \\
\hline yra / quira & pour les choses rondes (comme des oranges) \\
\hline ycha & pour les choses longues (comme des cierges) \\
\hline echu & pour les choses plates (comme du papier ou des tortillas) \\
\hline chere & pour les choses tissées (comme des draps) \\
\hline thumbi & $\begin{array}{l}\text { pour les choses comme des sacs, des sacoches, des } \\
\text { charges ou des régimes de bananes }\end{array}$ \\
\hline çutu & idem sauf pour les régimes de bananes \\
\hline xancha & pour les choses à quatre pieds (comme les animaux) \\
\hline chuиi & idem et aussi pour les poules et la volaille \\
\hline hutu & pour les choses comme les bottes ou les fagots \\
\hline anga & pour mettre une chose debout ou une chose dressée \\
\hline umba & pour les choses entassées (+ animé) \\
\hline tzuhti & pour deux choses entassées (+ animé) \\
\hline thsinguui & $\begin{array}{c}\text { pour les choses comme les plats, les écuelles, les tasses } \\
\text { lorsqu'ils sont mis les uns sur les autres }\end{array}$ \\
\hline para & pour les tasses (faites en écorce) lorsqu'elles sont séparées \\
\hline tacu & $\begin{array}{c}\text { pour les choses pliées ou plates et larges comme les livres } \\
\text { et les tapis en grande quantité (plus de un) }\end{array}$ \\
\hline $\begin{array}{l}\text { churu/ } \\
\text { chungua }\end{array}$ & pour les choses qui s'entassent sans ordre (- animé) \\
\hline
\end{tabular}

Les trois premiers éléments yra / quira, ycha et echu sont ceux qui sont encore utilisés aujourd'hui. Il semble donc que Gilberti a rangé ces unités par ordre de fréquence et de productivité. De plus, le sens de ces trois éléments n'est motivé que par une caractéristique physique de l'objet : [rond], [long] et [plat]. Au contraire, les autres unités forment un système complexe où 
différents traits interviennent : les éléments peuvent renvoyer à des qualités non inhérentes des entités (choses tissées), à des mesures (des tas ou des régimes), à des positions (des objets debout, empilés les uns sur les autres), à des caractères sémantiques comme l'animacité.

Actuellement, seules les trois premières unités existent, ce sont celles qui renvoient à la forme ou à des caractéristiques physiques des éléments référés. Les traits [rond], [long] et [plat] reprennent généralement les propriétés visibles, stables et inhérentes de l'objet référé par le nom (voir ci-dessous 3.4.).

\subsection{Erosion phonétique}

Les trois CNums peuvent apparaître dans le corpus avec leur forme pleine, constituée de la base et du suffixe nominalisateur kwa, par exemple echukwa, comme dans l'exemple (12). Ils forment un élément indépendant possédant son propre accent [echúkwa].

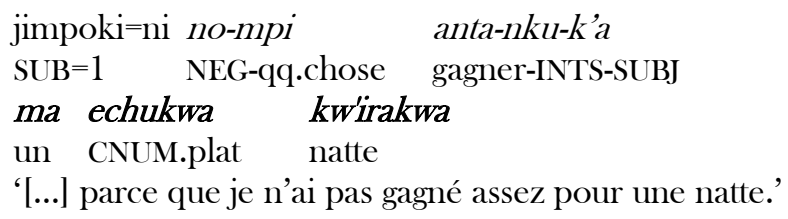

Néanmoins, dans de nombreux parlers, la forme attestée est réduite. Elle passe de trois syllabes à deux syllabes, comme en (13), et généralement à une syllabe, comme en (14). La conséquence de cette érosion phonétique est la dépendance formelle des CNums. Ils perdent leur accent et ne peuvent plus fonctionner comme des éléments libres, ils se suffixent donc au numéral qui les précédent. Dans ces contextes, le numéral perd souvent la dernière syllabe, comme en (14), ou la dernière voyelle, comme en (13).

\section{(13) tsiman-ichuk $=k^{\prime} u$}

deux-CNUM.plat=seulement

'Seulement deux' [forme complète : tsimani ichukwa] 


\section{(14) intsku- $\varnothing=r i n i$ tsima-chak kurhínta}

donner-IMP.2=1OBJ deux-CNUM.long pain

'Donne-moi deux pains.' [forme complète : tsimani ichakwa]

Ces érosions phonétiques ont pour conséquence la fusion du numéral et du CNum, ils sont formellement liés puisqu'un seul accent apparaît pour ces deux éléments. En effet, la forme complète comporte deux accents [tsimáni ichákwa], au contraire la forme réduite ne présente qu'un seul accent [tsimánichuk] ou [tsimáchak]. Ce processus révèle une variation diatopique de la langue. Selon les variétés, les CNums sont considérés comme des suffixes ou comme des éléments libres.

\subsection{Caractère optionnel}

Les CNums ne sont attestés dans notre corpus que dans 7\% des SNs avec un numéral. Dans 93\% des occurrences, le GN présente la forme [Num N], comme dans l'exemple (15a). Un même locuteur, dans un même discours peut d'ailleurs utiliser un CNum ou pas. L'énoncé en (15a) est prononcé trois énoncés après l'énoncé en $(15 b)$. Dans ce calcul, seuls sont pris en compte les numéros en purepecha de 1 à 5 et 10 puisque les CNums n’apparaissent qu'avec ces numéros. Ils ne sont jamais utilisés avec les numéros en espagnol, qui sont les plus nombreux puisque le purepecha a massivement emprunté les numéros à l'espagnol. Les Purepechas utilisent généralement les numéros de leur langue pour les nombres de 1 à 5 et pour 10, et les numéros de l'espagnol pour les autres. L'emprunt massif de numéros à l'espagnol a donc favorisé la réduction de l'utilisation des CNums.

\section{(15)a. tanimu achati-cha=nha ni-ra-s-ti}

trois homme-PL $=\mathrm{EV}$ aller-AOR-ASS3

'On dit que les trois hommes partirent.'

$\begin{array}{lll}\text { b. ima } & \text { tsimani echakwa } & \text { kwirípu-cha-ni } \\ \text { DEM } & \text { deux CNUM.long } & \text { personne-PL-OBJ } \\ \text { jinkoni } & \text { jama-s-ti } & \\ \text { COM } & \text { marcher-AOR-ASS3 } \\ \text { 'Il marchait avec les deux personnes.' }\end{array}$


Comme on vient de le voir l'utilisation des CNums est restreinte aux numéros en purepecha, elle l'est aussi à une quantité limitée de noms. En effet, les noms qui sont présents dans les SNs dans lesquels apparait un CNum appartiennent à la sphère quotidienne : des objets (natte, canoë), des aliments (tortilla, orange, pomme), des animaux, des êtres humains (voir 3.4.).

\subsection{Variations sémantiques}

Les CNums sont utilisés avec un nombre réduit d'une cinquante de noms différents, exprimant tous des objets, aliments, animaux ou êtres humains de la sphère quotidienne. Généralement, le CNum renvoie à la forme visible et inhérente du référent du nom. La forme echakwa 'long' est la plus fréquente, elle est associée aux noms désignant par exemple un canoë, une bûche, du bois, un arbre, une bougie, une banane, des animaux, des êtres humains. Le CNum echukwa 'plat' renvoie à une feuille, du papier, une table, une tortilla. Le troisième CNum, irhakwa 'rond' accompagne des entités comme une balle, une joue, une pierre, des aliments (orange, œuf, tomate, pomme, pomme de terre). Il est intéressant de signaler que l'utilisation des CNums avec des noms renvoyant à des êtres humains semble fluctuante puisque Friedrich (1970) montre des exemples comme nous le faisons alors que Vázquez Rojas (2012 : 89-92) affirme que cette utilisation est impossible. Dans son travail, Vázquez Rojas (2012 : 107-109) insiste aussi sur l'utilisation stricte des CNums en fonction de la propriété stable et permanente des objets.

Cependant, au-delà de cette division tripartite généralement respectée, on constate quelques utilisations contextuelles qui révèlent des variations d'utilisation en fonction de la perception du locuteur ou de l'utilisation de l'objet (Aikhenvald 2003 : 98, Friedrich 1970, Grinevald 1999, 2000). Dans mon corpus, j’ai pu observer quelques utilisations contextuelles. Par exemple, le nom qui désigne la femme est généralement accompagné par le CNum echakwa, cependant on peut aussi trouver le CNum irhakwa 'rond' afin d'insister sur la rondeur d'une femme particulière. Par ailleurs, un ballon de baudruche gonflé sera accompagné du CNum irhakwa 'rond' au contraire lorsqu'il n'est pas gonflé, le nom sera accompagné de echakwa 'long', manifestant ainsi la différence de 
forme en fonction de son état. Un dernier exemple d'utilisation contextuelle est celui de la natte qui peut être accompagnée du CNum echukwa 'plat' lorsqu'elle est étalée sur le sol, comme dans l'exemple (16). En revanche, le nom peut être accompagné par le CNum echakwa 'long' lorsque le locuteur réfère à l'objet tel qu'il est présenté chez le marchand (enroulé et posé verticalement sur le sol), comme dans l'exemple (17).
(16) jimpoki=ni no-mpi anta-nku-k'a
SUB $=1$ NEG-qq.chose gagner-INTS-SUBJ

\section{ma echukwa kwirakwa}
un CNUM.plat natte
'[...] parce que je n'ai pas gagné assez pour une natte.'

$\begin{array}{llll}\text { naxani } & \text { juka-para-ni } & \text { ma } & \text { echakwa } \\ \text { combien } & \text { mettre-épaule-NF } & \text { un } & \text { CNUM.long } \\ \text { kw'irakwa } & \text { yasï } & & \\ \text { natte } & \text { maintenant } & \\ \text { 'Combien coûte une natte maintenant?' } & \end{array}$

Le CNum echakwa 'long' est le plus fréquent, ce phénomène est explicable car les objets longs sont les plus fréquents et qu'il est associé aux animaux et aux êtres humains. Sa sphère d'utilisation est donc plus ample que celle des deux autres unités. Cette fréquence semble aussi due à une perte de motivation sémantique. Chamoreau (1999) et Monzón (1998) montrent que dans certains villages, respectivement à Jaracuaro et à Angahuan, l'adoption du CNum echakwa se réalise dans des contextes où la forme des objets requerrait un des deux autres CNums. Cette utilisation est systématique à Angahuan (Monzón 1998) alors qu'elle est fluctuante à Jaracuaro, comme on peut le constater dans les exemples (18) et (19). Le nom qui désigne la tortilla est le plus souvent accompagné du CNum ichukwa 'plat', comme en (18) mais le CNum ichakwa 'long' peut aussi être utilisé, comme en (19). Dans ce contexte, il ne peut s'agir d'une variation contextuelle (la tortilla pourrait être perçue comme longue lorsqu'elle est enroulée sur elle-même) puisque les tortillas étaient à plat empilées les unes sur les autres. De plus, l'utilisation de echakwa avec le nom désignant une tortilla, une feuille ou du papier est attestée dans 
d'autres exemples du corpus. Le CNum echakwa subit un processus de désémantisation, il ne réfère plus obligatoirement à une entité longue.
(18) ja-rha-s-ti
tsima-chuk
ichuskuta
être.là-FT-AOR-ASS3 deux-CNUM.plat tortilla
'Il y a deux tortillas.'

(19) intsku- $\varnothing=$ rini

donner-IMP.2=1OBJ

ma-chak

ichuskuta

un-CNUM.long tortilla

'Donne-moi une tortilla.'

L'utilisation croissante de echakwa est aussi attestée avec des noms abstraits. Ces noms ne présentent pas de forme physique particulière qui motive l'utilisation d'un CNum en particulier. Lorsqu'un CNum est présent dans le GN, il s'agit toujours de echakwa. Dans le corpus, echakwa peut apparaître avec les termes wantakwa 'récit' et palabra 'mot', comme dans l'exemple (20) :

\begin{tabular}{llll} 
(20) tsimani o & \multicolumn{2}{l}{ tanimu repetiri-ku-ni } \\
deux ou & trois répéter-APPL3-NF \\
ma echakwa & palabra-ni & para ji \\
un CNUM.long & mot-OBJ & pour 1IND \\
u-ni ya & sesi & \\
pouvoir-NF déjà & bien & \\
'Répéter deux ou trois, un mot pour que je le fasse bien.'
\end{tabular}

La perte de vitalité des CNums a été constatée à différents niveaux développés ci-dessus révélant un système menacé de disparition. J'avais déjà fait ce constat il y a près de 15 ans après un premier diagnostic de la dynamique de ces éléments effectué dans le village de Jaracuaro où les CNums ne sont presque plus employés (Chamoreau 1999). Or le recueil de données dans de nombreux villages permet d'appréhender un changement peu perceptible dans les quelques exemples recueillis à Jaracuaro. Si au niveau sémantique, les CNums montrent des variations et des pertes de motivation, l'utilisation de ces unités dévoile une motivation pragmatique particulière : leur utilisation restreinte se limite à des contextes où le référent du nom est prominent. 


\section{MOTIVATION PRAGMATIQUE}

L'utilisation des CNums en purepecha individualise l'entité quantifiée, ils ne désignent pas un ensemble d'entités et n'ont pas de statut compositionnel (Vázquez Rojas 2012 : 112), comme on peut l'observer en (21). En effet, bien que la composition de ces trois étoiles pourrait former une ligne, il n'est pas possible d'utiliser le CNum ichakwa 'long'. Le CNum qui est utilisé correspond à la forme de chaque étoile, c'est-à-dire irhakwa 'rond', comme en (22).

(21) * awánta-rhu
ciel-LOC

era-nchi-kurhi-xa-ti

voir-haut-REF-PROG-ASS3

$\begin{array}{lll}\text { tanímu } & \text { ichákwa } & \text { jóskw-icha }=k \text { 'u } \\ \text { trois } & \text { CNUM.long } & \text { étoile-PL=seulement }\end{array}$

'On voit les trois étoiles seulement dans le ciel, en ligne.'

(22) awánta-rhu

era-nchi-kurhi-xa-ti

ciel-LOC

voir-haut-REF-PROG-ASS3

tanímu

trois

erhákwa

jóskw-icha $=k^{\prime} u$

'On voit les trois étoiles seulement dans le ciel.'

Au-delà de cette caractéristique sémantique qui montre un emploi individualisateur, on observe que leur utilisation se réalise dans des contextes limités, spécifiques et marqués. L'analyse des occurrences des CNums montre qu'ils apparaissent dans des contextes où l'entité référée est identifiable et prominente. Lambrecht (1994: 77-78) définit un référent identifiable comme celui dont la représentation est partagée par le locuteur et l'interlocuteur. Il peut être accessible dans l'esprit des interlocuteurs car sa connaissance est antérieure au moment de l'interlocution ou bien parce qu'il est physiquement accessible au moment de l'interlocution. Dans les deux cas, son identifiabilité rend prominente dans le discours l'entité individuée, c'est-à-dire qu'elle culmine au-dessus des éléments alentours ou s'en démarque. Elle constitue ainsi le plus souvent le premier plan de la situation, immédiatement accessible aux interlocuteurs. La notion d'identifiabilité telle qu'elle est appréhendée par Lambrecht (1994) 
est proche de la notion de familiarité utilisée par exemple par Roberts (2003).

La présence d'un CNum dans une conversation ou un récit apporte des informations pragmatiques en indiquant la prominence de l'entité désignée. Dans l'exemple (25), deux femmes parlent des achats qu'elles ont effectués au marché, elles énumèrent et comparent les fruits et légumes qu'elles ont achetés. Dans la grande majorité de la conversation aucun CNum n'est utilisé, comme en (25). Dans cet exemple, on peut observer une distinction de définitude opérée par la marque de pluriel : la présence du pluriel sur le terme naraxa-icha 'oranges' indique que les aliments sont conçus comme définis et individualisables, au contraire, son absence sur le terme k'awaxï 'piments' montre qu'ils sont appréhendés comme indéfinis. A un moment, une des femmes évoque quatre courgettes achetées la veille en compagnie de sa commère, courgettes appréciées par son mari. Elle fait référence à ces quatre courgettes en utilisant un CNum, comme on peut l'observer en (26). C'est le seul CNum présent dans cette conversation où on compte treize GNs avec un numéral. L'utilisation du CNum dans l'énoncé en (26) met en valeur un référent identifiable, qui se démarque des autres, particulièrement important et prominent pour ces deux femmes qui expliquent qu'elles les avaient choisis avec soin. On peut observer que le terme purhu 'courgette' est modifié par le démonstratif tsï et le pluriel, ce qui permet une perception définie et individualisée des aliments, que renforce la présence du CNum.

\section{(25) exe-i tsimani naraxa-icha, tanimu k'awaxi voir-IMP2 deux orange-PL trois piment 'Regarde les deux oranges, trois piments [...]'}

(26) Puki arha-x-ti tsï-ni t’ám-ichak purhu-icha

Puki ingérer-AOR-ASS3 dem-obj quatre-CL.long courgette-PL 'Puki a mangé ces quatre courgettes.'

Cette motivation pragmatique est particulièrement observable avec le numéral ma 'un' qui apparait dans 43\% des GNs dans lesquels un CNum est utilisé. En purepecha, la forme ma renvoie tant au numéral 'un' qu'à l'article indéfini, ce dernier étant une 
forme grammaticalisée du premier (Chamoreau 2012). La présence d'un CNum permet parfois de distinguer entre l'article indéfini, comme en (27) et le numéral, comme en (28) en indiquant que la quantification est pertinente. Cependant, le plus souvent le rôle de l'introduction du CNum est d'indiquer la prominence pragmatique de l'entité référée, comme en (29). Dans les énoncés en (28) et (29), la présence du CNum permet de souligner l'importance de l'entité introduite dans le GN et de la démarquer d'autres : un des deux voleurs introduits antérieurement dans le récit en (28) et une des quatre autres pièces de la maison présentées avant, en (29).

$\begin{array}{lll}\text { ja-rha-s-p-t } i=n h a & \text { ma } & \text { achati } \\ \text { etre.là-FT-AOR-PAST-ASS3=EV } & \text { ART.INDEF } & \text { homme } \\ \text { enka } \quad n i-p-k a & \text { ireta jimpo } & \\ \text { SUB aller-AOR.PAST-SUBJ } & \text { village INST } & \\ \text { 'On avait dit qu'il y avait homme qui partit au village } & \text { [...]' }\end{array}$

(28) jimak’ani ma echakwa sïpaphiri tsí-nharhi-ni alors un CNUM.long voleur réveiller-visage-NF 'Alors, un voleur (un, pas les deux) se réveilla [...]'

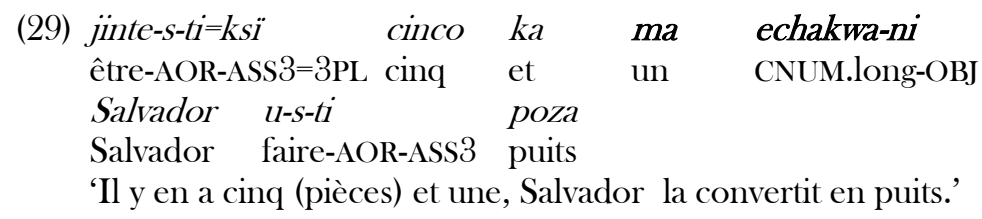

\section{REFLEXIONS FINALES}

Selon Grinevald (2000 : 85-86), le processus de perte des classificateurs se manifeste par l'appauvrissement de l'inventaire des unités et la simplification de la catégorisation plus que par la perte de la construction elle-même. Cette analyse sied particulièrement bien à la situation actuelle des CNums en purepecha, puisque la construction subsiste bien qu'il existe une restriction de l'inventaire, une érosion phonétique, une utilisation peu fréquente et optionnelle, des variations et désémantisations sémantiques. Néanmoins, le purepecha montre une forte originalité puisque si l'utilisation du CNum au sein d'un GN a le plus souvent conservé 
une motivation sémantique (caractéristique physique et individualisation de l'entité), elle a innové en ayant développé une nouvelle fonctionnalité pragmatique (prominence et identificabilité de l'unité).

\section{Abréviations utilisées}

$\begin{array}{llll}\text { AOR } & \text { aoriste } & \text { INTS } & \text { intensificateur } \\ \text { APPL } & \text { applicatif } & \text { LOC } & \text { locatif } \\ \text { ART } & \text { article } & \text { NEG } & \text { négation } \\ \text { ASS } & \text { assertif } & \text { NF } & \text { non-fini } \\ \text { COM } & \text { comitatif } & \text { OBJ } & \text { objectif } \\ \text { CNUM } & \text { classificateur nominal } & \text { PAST } & \text { passé } \\ \text { DEM } & \text { démonstratif } & \text { PL } & \text { pluriel } \\ \text { EV } & \text { évidentiel } & \text { PRED } & \text { prédicativisateur } \\ \text { FT } & \text { formatif } & \text { PROG } & \text { progressif } \\ \text { IMP } & \text { impératif } & \text { REF } & \text { réfléchi } \\ \text { IND } & \text { indépendant } & \text { SN } & \text { syntagme nominal } \\ \text { INDEF } & \text { indéfini } & \text { SUB } & \text { subordonnant } \\ \text { INT } & \text { interrogatif } & \text { SUBJ } & \text { subjonctif }\end{array}$

\section{Références bibliographiques}

AIKHENVALD Alexandra, 2003, Classifiers. A Typology of Noun Categorization Devices, Oxford, Oxford University Press.

CAPISTRÁN Alejandra, 2000, "Verbos clasificatorios en p'orhépecha. Expresión de relaciones espaciales", in M. del Carmen MORÚA LEYVA et G. LÓPEZ CRUZ, V Encuentro Internacional de Lingüística en el Noroeste II, Hermosillo, UniSon, pp. 327-344.

CHAMOREAU Claudine, 1999, "Evolution des indices catégoriels en phurhépecha”, Faits de Langues 14, pp. 143-152.

CHAMOREAU Claudine, 2009, Hablemos purepecha. Wantee juchari апари, México, UIIM / IIH-UMSNH / IRD / Ambassade de France au Mexique CCC-IFAL/ Grupo Kw'anískuyarhani.

CHAMOREAU Claudine, 2012, "Dialectology, typology, diachrony and contact linguistics. A multi-layered perspective in Purepecha”, STUF, Language Typology and Universals 65, 1, pp. 6-25.

CHAMOREAU Claudine et ARGUETA Arturo, 2011, "Lo animado y lo inanimado entre los P'urhépecha de Michoacán" in Perig PITROU, María del Carmen VALVERDE VALDÉS et Johannes NEURATH (eds), La noción de vida en Mesoamérica, Mexico, CEMCA / UNAM, pp. 95-117. 
FRIEDRICH Paul, 1970, "Shape in grammar", Language 46, 2. pp. 379-407.

FRIEDRICH Paul, 1984, "From meaning to sound", Handbook of Middle American Indians 2, Austin, University of Texas Press, pp. 56-82.

GILBERTI Maturino, 1987 [1558], Arte de la lengua de Michuacan, Morelia, Fimax.

GREENBERG Joseph, 1974, "Numeral classifiers and substantival number : problems in the genesis of a linguist type, in Proceedings of the $11^{\text {th }}$ International Congress of Linguistics, Bologna, pp. 17-37;

GRINEVALD Colette, 1999, "Typologie des systèmes de classification nominale" Faits de Langues 14, pp. 101-122.

GRINEVALD Colette, 2000. "A morphosyntactic typology of classifiers", G. SENFT (ed.), Systems of Nominal Classification, Cambridge, Cambridge University Press, pp. 50-92.

HEINE Bernd, 1997, Cognitive Foundations of Grammar, Oxford, Oxford University Press.

HEINE Bernd, 2012, "On polysemy copying and grammaticalization in language contact”, in Claudine CHAMOREAU y Isabelle LÉGLISE (eds), Dynamics of Contact-Induced Language Change, Berlin, Mouton de Gryuter, pp. 125-166.

LAGUNAS Juan Baptista de, 1983 [1574], Arte y Dictionario con otras obras en lengua Michuacana, Morelia, Fimax.

LAMBRECHT Knud, 1994, Information Structure and Sentence Form, Cambridge, Cambridge University Press.

MONZÓN Cristina, 1998, "Variantes dialectales del p’urhépecha", Julio CALVO-PÉREZ et Daniel JORQUES JIMÉNEZ (eds), Estudios de lengua y cultura amerindias II: lenguas, literaturas, Valencia, Universidad de valencia, pp. 61-83.

NAVA Fernando, 1997, "Los clasificadores numerales del p'urhépecha prehispánico”, Anales de Antropología 31, pp. 299-309.

ROBERTS Craige, 2003, "Uniqueness in definite noun phrases", Linguistics and Philosophy 26, pp. 287-350.

VÁZQUEZ ROJAS Violeta, 2012, "The Syntax and Semantics of Purépecha Noun Phrases and the Mass/Count Distinction”. PhD Dissertation. New York University. 